\title{
Mechanisms of $\mathrm{CCl}_{4}$-induced liver fibrosis with combined transcriptomic and proteomic analysis
}

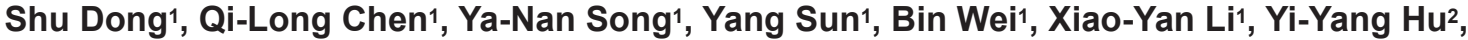 \\ Ping Liu ${ }^{3}$ and Shi-Bing Su ${ }^{1}$
}

\author{
${ }^{1}$ Research Center for Traditional Chinese Medicine Complexity System, Shanghai University of \\ Traditional Chinese Medicine, Shanghai, 201203, China \\ 2Institute of Liver Diseases, Shuguang Hospital Affiliated to Shanghai University of \\ Traditional Chinese Medicine, Shanghai 201203, China \\ ${ }^{3}$ Institute of E, Shanghai University of Traditional Chinese Medicine, Shanghai 201203, China
}

(Received February 16, 2016; Accepted June 5, 2016)

\begin{abstract}
The classic toxicity of carbon tetrachloride $\left(\mathrm{CCl}_{4}\right)$ is to induce liver lesion and liver fibrosis. Liver fibrosis is a consequence of chronic liver lesion, which can progress into liver cirrhosis even hepatocarcinoma. However, the toxicological mechanisms of $\mathrm{CCl}_{4}$-induced liver fibrosis remain not fully understood. We combined transcriptomic and proteomic analysis and biological network technology, predicted toxicological targets and regulatory networks of $\mathrm{CCl}_{4}$ in liver fibrosis. Wistar rats were treated with $\mathrm{CCl}_{4}$ for 9 weeks. Histopathological changes, hydroxyproline (Hyp) contents, serum ALT and AST in the $\mathrm{CCl}_{4}$-treated group were significantly higher than that of $\mathrm{CCl}_{4}$-untreated group. $\mathrm{CCl}_{4}$-treated and -untreated liver tissues were examined by microarray and iTRAQ. The results showed that 3535 genes (fold change $\geq 1.5, P<0.05$ ) and 1412 proteins (fold change $\geq 1.2, P<0.05$ ) were differentially expressed. Moreover, the integrative analysis of transcriptomics and proteomics data showed 523 overlapped proteins, enriched in $182 \mathrm{GO}$ terms including oxidation reduction, response to oxidative stress, inflammatory response, extracellular matrix organization, etc. Furthermore, KEGG pathway analysis showed that 36 pathways including retinol metabolism, PPAR signaling pathway, glycolysis/gluconeogenesis, arachidonic acid metabolism, metabolism of xenobiotics by cytochrome P450 and drug metabolism. Network of protein-protein interaction (PPI) and key function with their related targets were performed and the degree of network was calculated with Cytoscape. The expression of key targets such as CYP4A3, ALDH2 and ALDH7A1 decreased after $\mathrm{CCl}_{4}$ treatment. Therefore, the toxicological mechanisms of $\mathrm{CCl}_{4}$-induced liver fibrosis may be related with multi biological process, pathway and targets which may provide potential protection reaction mechanism for $\mathrm{CCl}_{4}$ detoxication in the liver.
\end{abstract}

Key words: Liver fibrosis, $\mathrm{CCl}_{4}$, Toxicity, Transcriptomics, Proteomics, Integrative analysis

\section{INTRODUCTION}

Liver fibrosis is a common process that occurs in response to kinds of liver injuries such as toxic or drugs exposure (Duval et al., 2015). It is characterized by the excess deposition of extracellular matrix (ECM) components which often lead to hepatic dysfunction, even hepatocellular carcinoma (Li et al., 2015b). Liver fibrosis is an important public health concern which can progress to cirrhosis, portal hypertension, and hepatocellular carcinoma (HCC), leading to increased morbidity and mor- tality (Enomoto et al., 2014). Among all reasons inducing liver injury and fibrosis, toxic reagents are one that cannot be ignored. The causes of drug-induced liver injury are multifactorial, including toxic effects caused by reactive metabolites, reactive oxygen species, inflammatory reactions, and imbalances between cellular damage and protective responses (Xuan et al., 2015) which will lead to inflammation, oxidative stress induction and fibrosis in the liver (El-Bini et al., 2015). Carbon tetrachloride $\left(\mathrm{CCl}_{4}\right)$, a commonly used laboratory reagent marked by its toxicity leading to liver lesion and liver fibrosis, has

Correspondence: Shi-Bing Su (E-mail: shibingsu07@163.com) 
been extensively applied in liver-related studies (Huang et al., 1998). Previous studies showed that in rats treated with $\mathrm{CCl}_{4}$ for eight weeks, lipid profile, liver enzymes and oxidative stress markers increased remarkably, total protein and high-density lipoprotein (HDL) decreased dramatically (Ebeid et al., 2015). Microscopic examination of the treated rats exhibited fatty changes along with the increase of inflammatory collections, the loss of normal hepatocytes, obvious collagen deposition and fiber segmentation formation (Dong et al., 2015). The chronic toxicity caused by $\mathrm{CCl}_{4}$ also includes the cardiac function test, significantly decreased the activities of antioxidant enzymes and glutathione contents whereas significant increase was found in lipid peroxidation. Moreover, $\mathrm{CCl}_{4}$ induction in rats caused DNA fragmentation and histopathological abnormalities (Sahreen et al., 2014). However, the toxicological mechanisms remain not fully understood.

The rapid progress of systems biology and bioinformatics has provided not only a systems-level understanding of biological processes and disease complexity but also an efficient and promising approach, such as network analysis (Kong et al., 2015). In studies of transcriptomics, microarray technology is a widely used approach because of its easy, high-throughput data generation and affordable cost (Osada, 2013). Isobaric tags for relative and absolute quantitation (iTRAQ), a high-throughput detection technology for measuring expression levels of different proteins has been widely used. All these systems biology and high-throughput detection technologies provide a more complete picture of complex biological processes, promote the understanding of the toxicological mechanisms of toxic reagents and will further provide a detoxification or more pointed treatment (Meierhofer et al., 2014).

In the present study, for better understanding the toxicological mechanisms of $\mathrm{CCl}_{4}$ toxicity in inducing liver lesion and fibrosis, we performed integrative analysis with mRNA microarray and iTRAQ data to evaluate the significance of alterations of biological functions and associated signal pathways, which are potentially involved in liver lesion and fibrosis induced by $\mathrm{CCl}_{4}$.

\section{MATERIALS AND METHODS}

\section{Animals and $\mathrm{CCl}_{4}$ administration}

All experiments in this study were approved by the Animal Ethics Committee (Research Center of Animal Experiments, Shanghai University of Traditional Chinese Medicine) and performed in accordance with The Guide for Animal Care and Use. Wistar rats weighing 150-160 g were purchased from the Shanghai Laboratory
Animal Center of the Chinese Academy of Sciences (Shanghai, China). $\mathrm{CCl}_{4}$ (batch number: 20070721) and olive oil (batch number: 060312) was purchased from Sinopharm Co., Ltd (Shanghai, China). The rats were acclimatized breeding to laboratory conditions for 7 days. Then all these rats were allocated to two groups: $\mathrm{CCl}_{4}$-untreated group $(\mathrm{N}=6)$ and $\mathrm{CCl}_{4}$-treated group $(\mathrm{N}=8)$ by a random sampling method based on bodyweight. The rats in the CCl4-untreated group were treated with conventional breeding and those in the $\mathrm{CCl}_{4}$-treated group were intraperitoneally injected with $50 \% \mathrm{CCl}_{4}$ olive oil solution at $1 \mathrm{~mL} / \mathrm{kg}$ body weight twice a week (every Monday and Thursday) for 9 weeks. Daily observation of clinical symptoms was conducted throughout the study. Body weight was measured twice a week.

\section{Samples and parameters}

After 9 weeks administration, the rats were fasted overnight and anesthetized after weighing. Blood was collected via the abdominal aorta under anesthesia. For liver function examination, aspartate aminotransferase (AST) and alanine aminotransferase (ALT) were determined with biochemical kits which were purchased from Nanjing-Jiancheng Biological Engineering Research Institute (Nanjing, China). The significance of these changes was calculated using Student's t-test. Differences for each parameter (excluding general conditions) were evaluated and considered to be significant at $p$-values less than 0.05 .

At termination, liver tissues were collected, weighed, and cut into small pieces. The liver tissues big as $0.5 \mathrm{~cm}^{2}$ were fixed in $4 \%$ paraformaldehyde phosphate buffer solution and subjected to paraffin embedding, then for hematoxylin and eosin (H\&E) staining and sirius red staining, and microscopic examination. Histopathological changes of liver fibrosis were evaluated by the five grades (0, I, II, III, IV). Hydroxyproline (Hyp) contents of liver tissues were also tested with Hyp-detection kit (Nanjing-Jiancheng). The remaining liver tissues were frozen in liquid nitrogen and preserved at $-80^{\circ} \mathrm{C}$ in a freezer. Liver tissues from 3 animals of each group were selected for transcriptomic and proteomic analysis.

\section{Total RNA extractions and mRNA microarrays assay}

Total RNA was isolated from liver tissue of the same rats subjected to proteome analysis using TRIZOL Reagent (Cat\#15596-018, Life Technologies, Carlsbad, CA, USA) following the manufacturer's instructions and checked for a RIN number to inspect RNA integration by an Agilent Bioanalyzer 2100 (Agilent Technologies, Santa 
Clara, CA, USA). Qualified total RNA was further purified by RNeasy mini kit (Cat\#74106, QIAGEN, GmBH, Germany) and RNase-Free DNase Set (Cat\#79254, QIAGEN) and then were stored at $-80^{\circ} \mathrm{C}$. A Quality Control was carried out with NanoDrop ND-1000 (NanoDrop Technologies, Inc., 3411 Silverside Road, Bancroft Building, Wilmington, DE 19810, USA).

MRNA microarray analysis was conducted on each total RNA sample using Low Input Quick Amp Labeling Kit, One-Color (Cat\#5190-2305, Agilent Technologies) and Gene Expression Hybridization Kit (Cat\#5188-5242, Agilent Technologies). The procedure was conducted basically following the manufacturer's protocol. Slides were scanned by Agilent Microarray Scanner (Cat\#G2565CA, Agilent Technologies) with default settings. Feature Extraction software 10.7 (Agilent Technologies) was used to get the raw data and then it was normalized by Quantile algorithm, Gene Spring Software 11.0 (Agilent Technologies).

\section{Protein preparation and iTRAQ quantification}

Frozen samples were ground in liquid nitrogen to a fine powder and suspended in STD buffer (4\% SDS, $1 \mathrm{mM}$ DTT, $150 \mathrm{mM}$ TrisHCl pH8.0). The suspension was vortexed and kept in boiling water for 5 min, then centrifuged for supernatant after ultrasonication. The protein concentration of each sample was determined using a 2D Quantification kit (GE Healthcare, Buckinghamshire, UK). A total of $100 \mu \mathrm{g}$ of each sample was denatured, reduced and alkylated as described in the iTRAQ protocol (Applied Biosystems, Foster City, CA, USA). Each sample was digested with $0.1 \mu \mathrm{g} / \mu \mathrm{L}$ trypsin solutions at $37^{\circ} \mathrm{C}$, overnight. The digested peptides were dried by vacuum centrifugation.

$\mathrm{CCl}_{4}$-untreated and $\mathrm{CCl}_{4}$-treated samples were respectively labeled with 113 and 117 iTRAQ tags according to the manufacturer's protocol (Applied Biosystems, USA). The six samples were pooled and vacuum-dried. The pooled sample was separated on the Poly-LC strong cation exchange column $(4.6 \times 100 \mathrm{~mm})$ on a Nano HPLC System (GE Healthcare). Subsequently, the fractionated samples were analyzed by LC-MS/MS based on Q-Exactive mass spectrometer (Thermo Finnigan, San Jose, CA, USA).

For peptide data analysis, raw mass data was processed using MASCOT software (Proteomics Tools) and normalized according to the signal values. Proteins were finally identified with the following filters (Protein FDR $\leq 0.01$; Peptide FDR $\leq 0.01$ ).

\section{Quantitative RT-PCR (qRT-PCR)}

The extracted $2 \mu \mathrm{g}$ total RNA was used for reverse transcription (RT) to generate template cDNA. The relative mRNA levels were determined with quantitative PCR. Since the expressions of PPIA in all samples were much more stable compared to GAPDH, the PPIA was served as an internal reference gene. The PCR parameters were as follows: $95^{\circ} \mathrm{C}$ for $1 \mathrm{~min}$ followed by 40 cycles of denaturation at $95^{\circ} \mathrm{C}$ for $15 \mathrm{sec}$, annealing at $60^{\circ} \mathrm{C}$ for $15 \mathrm{sec}$ and extending at $72^{\circ} \mathrm{C}$ for $45 \mathrm{sec}$. The primer sequences were designed using Primer 5.0 and were searched for specificity with NCBI-Blast. The primer sequences are as follows: CYP4A3: (F) 5'- ATA CCC AAA GGT ATC ACA ACC AC-3' and (R) 5'- ATG GCT ATG GCG AGG AGA ATC-3'; Cyp3a18: (F) 5'- ATC CCT TCG TGC AGA AAG CC-3' and (R)5'- CTT TTG CAC ATT GGG GCG AG-3'; ALDH7A1: (F) 5'- GTT GGA GCT CGG AGG AAA CA-3' and (R)5'-TTC GTG CAA AAA CAG TCG CC-3'; ALDH2: (F) 5'- TGG CTG ATC TCA TCG AAC GG-3' and (R)5'-CCA GCC AGC ATA ATA GCG GA-3'; and PPIA: (F) 5'- CCA AAC ACA AAT GGT TCC CAG T -3' and (R) 5'- ATT CCT GGA CCC AAA ACG CT -3'. The quantitative PCR results were calculated using the $2-\Delta \Delta C t$ method.

\section{Western blot}

Liver tissue homogenates were sonicated to dissolve completely and then centrifuged at $12,000 \mathrm{rpm}$ for $30 \mathrm{~min}$ at $4^{\circ} \mathrm{C}$ to separate the membrane-containing fraction (pellet) from the cytosol. Proteins $(100 \mu \mathrm{g})$ were separated by $10 \%$ SDS-polyacrylamide gel electrophoresis. The separated proteins were blotted onto nitrocellulose membrane (NC membrane, Millipore, Billerica, MA, USA), and then the membrane was washed for 10 min with TBST and immersed in blocking buffer containing 5\% nonfat dry milk in TBST for $1 \mathrm{hr}$ at room temperature. The blot was washed with TBST and finally incubated overnight at $4{ }^{\circ} \mathrm{C}$ with polyclonal primary antibodies CYP4A3 (Abcam, Cambridge, UK), diluted 1:2000, ALDH2 (Abcam) and ALDH7A1 (Proteintech Group, Inc., Chicago, IL, USA), diluted 1:500 and Cyp3a18 (Shanghai-Youke Biotechnology, Inc., Shanghai, China), diluted 1:1000 in 5\% nonfat dry milk. Then, the blot was incubated with secondary antibody (Cell Signaling Technology, Danvers, MA, USA) for $1 \mathrm{hr}$ at room temperature. Finally, the blot was scanned by infrared rays with the Odyssey Infrared Imager(LI-COR, Lincoln, NB, USA), and bands were quantified by densitometry using Odyssey software (version 1.2, LI-COR). 


\section{Data analysis}

In order to evaluate the key proteins involved in toxic effects of $\mathrm{CCl}_{4}$, we performed venny analysis to screen the overlapped proteins based on differentially expressed genes (DEGs) and differentially expressed proteins (DEPs). Differential mRNA expression between $\mathrm{CCl}_{4}{ }^{-}$ treated and $\mathrm{CCl}_{4}$-untreated tissues was analyzed using SAS online analysis tool and the mRNAs with a fold change| more than 1.5 and p-value less than 0.05 were considered to be DEGs. And differential protein expression between $\mathrm{CCl}_{4}$-treated and $\mathrm{CCl}_{4}$-untreated tissues was analyzed using fold change of protein expression profile, and the proteins with a |fold change| more than 1.5 were considered to be DEPs.

The gene ontology (GO) and pathway involved with these overlapped proteins were further analyzed using DAVID online analysis and the parameters were set as follows: count $=5$, ease $=0.05$. We built a protein-protein interaction network of these overlapped proteins using STRING online analysis and Cytoscape version 3.2.0 to evaluate the much more important proteins among all those overlapped proteins. The degree of the network was computed and the proteins with degrees higher than mean degree were considered to have much more close relationship with toxicity of $\mathrm{CCl}_{4}$. Network of key function with their related targets were also constructed to evaluate the important GO terms, pathways and targets. Based on all these analysis results, we further concluded the molecule mechanism of toxicity of $\mathrm{CCl}_{4}$.

\section{RESULTS}

\section{Liver damage induction}

In this study, we performed regular observation of signs of rats in the $\mathrm{CCl}_{4}$-untreated and $\mathrm{CCl}_{4}$-treated groups. During the nine weeks, rats in the $\mathrm{CCl}_{4}$-untreated group were in good condition. As for the rats in the $\mathrm{CCl}_{4}$-treated group, during the first 4 weeks, their body weight kept on reducing with poor spirit and appetite. From the fifth week, their weight only slightly decreased. Body weights and the weight ratio of liver/body are presented after all the rats were sacrificed. Trend toward decrease in body weight and increase in weight ratio of liver/body with statistical significance was induced by administration of $\mathrm{CCl}_{4}$ as compared to the $\mathrm{CCl}_{4}$-untreated group (Figs. 1A, 1B).
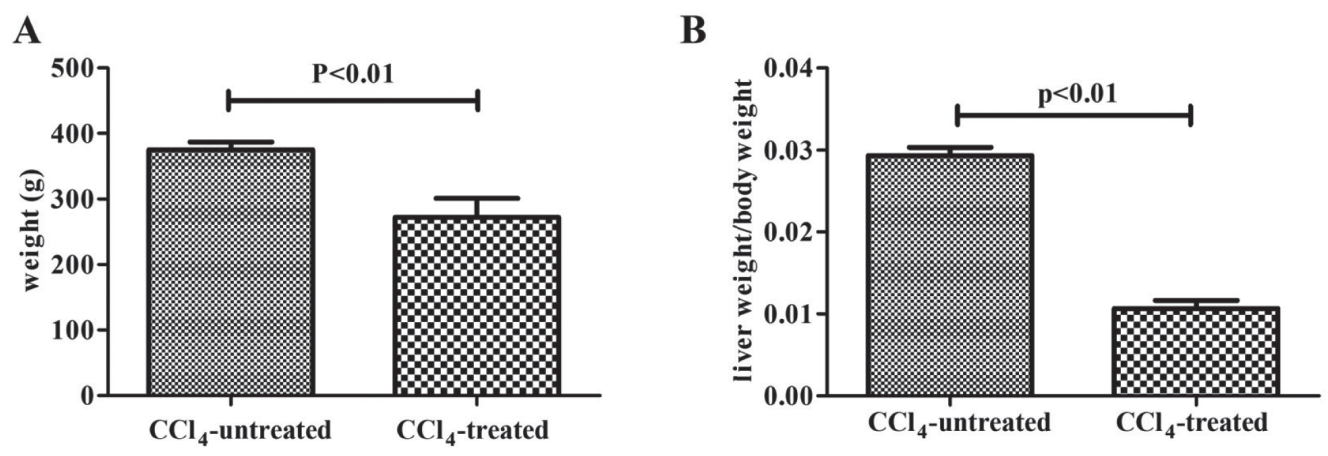

C

D

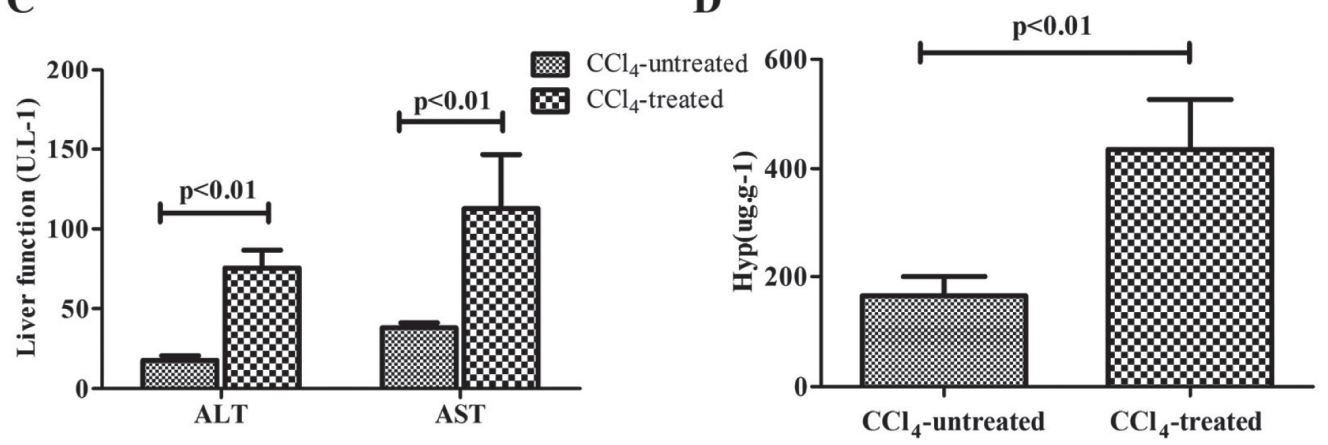

Fig. 1. Body weight, ratio of liver/body weight, serum ALT, AST and Hyp contents in rats. A, Body weight; B, weight ratio of liver/body; C, serum ALT and AST; D, Hyp contents in $\mathrm{CCl}_{4}$-untreated and $\mathrm{CCl}_{4}$-treated group. $\mathrm{CCl}_{4}$-untreated group $(\mathrm{N}=6)$, CCl4-treated group $(\mathrm{N}=8)$. 
Mechanisms of $\mathrm{CCl}_{4}$-induced liver fibrosis

Blood biochemistry examination was also performed. ALT and AST significantly increased in the $\mathrm{CCl}_{4}$-treated group as compared with the $\mathrm{CCl}_{4}$-untreated group which showed evident toxicity in inducing inflammation in $\mathrm{CCl}_{4}$-treated rats (Fig. 1C).

\section{Fibrosis induction}

Histopathological examination, fibrosis grading and examination of the content of Hyp in the rat livers were performed to evaluate the toxicity of $\mathrm{CCl}_{4}$ in fibrosis induction.

HE staining of rats in the CCl4-untreated group showed that the hepatic lobule structure is clear and no inflammatory cells. After nine weeks of $\mathrm{CCl}_{4}$ administration, moderately developed inflammatory cell infiltration around liver cells was detected in the livers of all rats treated with $\mathrm{CCl}_{4}$ which were in accordance with the results of liver function (Fig. 2A). Sirius red staining showed that there was no fibrosis in rats of the $\mathrm{CCl}_{4}$-untreated group, while in the $\mathrm{CCl}_{4}$-treated group, the structure of liver lobule was destroyed by paraplastic connective tissue and mild to serious fibrosis was detected (Fig. 2B). Fibrosis grades were further evaluated based on the histopathological examination and the results showed that in the CCl4untreated group, there were 6 rats were of fibrosis grade 0 , which meant none of them had fibrosis. In the $\mathrm{CCl}_{4}$ administration group, 3 rats were of fibrosis grade III, 4 rats of fibrosis grade IV and 1 rat of fibrosis grade V, which meant that rats in the $\mathrm{CCl}_{4}$-treated group all had liver fibrosis (Table 1). Another parameter to evaluate the liver fibrosis degree is the content of Hyp in liver tissue. Compared with the CCl4-untreated group, Hyp contents in the $\mathrm{CCl}_{4}$-treated group were significantly increased (Fig. 1D).

\section{Overlapped DEGs and DEPs}

In this study, expression profiles of mRNAs in $\mathrm{CCl}_{4}$ induced liver fibrosis were assessed using microarray analysis. Differentially expressed genes (DEGs) were calculated with SAS and then screened with a fold change $\geq 1.5$ and $\mathrm{p}$ value $\leq 0.05$. Compared with the $\mathrm{CCl}_{4}$-untreated group, 3535 genes were differentially expressed, of which 1474 genes were down-regulated and 2061 were up-regulated. In quantitative iTRAQ-coupled LC-
$\mathbf{A}$

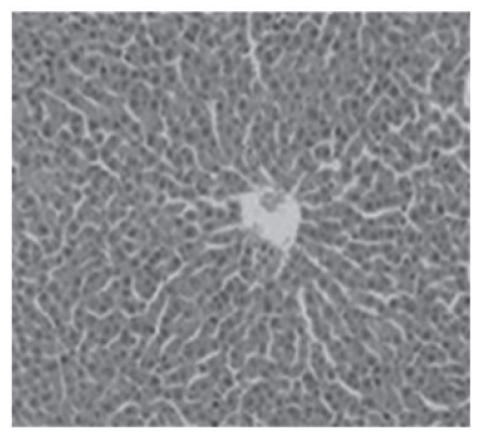

$\mathrm{CCl}_{4}$-untreated

B

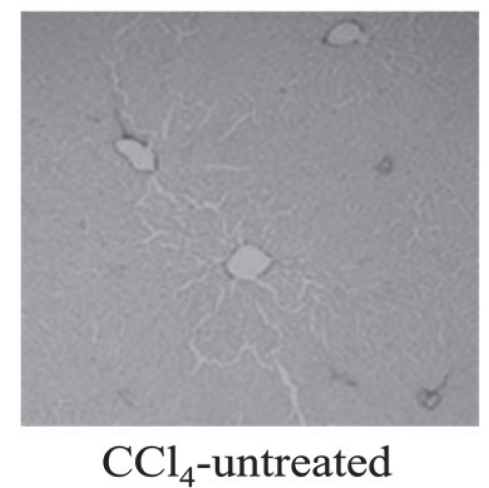

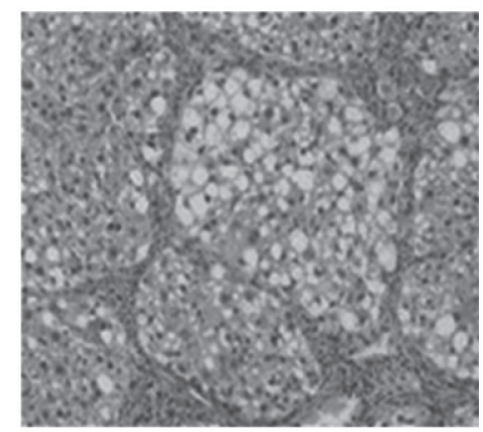

$\mathrm{CCl}_{4}$-treated

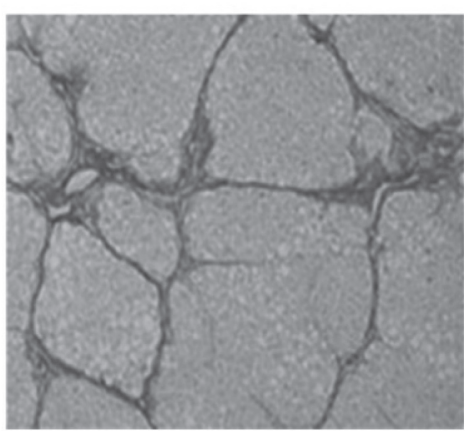

$\mathrm{CCl}_{4}$-treated

Fig. 2. Histopathological examinations in $\mathrm{CCl}_{4}$-untreated and $\mathrm{CCl}_{4}$-treated rats. $\mathrm{A}$, $\mathrm{HE}$ staining $(10 \times 20)$; $\mathrm{B}$, sirius red staining $(10 \times 10)$. 
Table 1. Fibrosis grades based on histopathological examination.

\begin{tabular}{llllccc}
\hline \multirow{2}{*}{ Group } & $\mathrm{n}$ & \multicolumn{5}{c}{ Fibrosis grades (cases) } \\
\cline { 3 - 7 } & & 0 & $\mathrm{I}$ & $\mathrm{II}$ & $\mathrm{III}$ & IV \\
\hline $\mathrm{CCl}_{4}$-untreated & 6 & 6 & 0 & 0 & 0 & 0 \\
$\mathrm{CCl}_{4}$-treated & 8 & 0 & 0 & 3 & 4 & 1 \\
\hline
\end{tabular}

MS/MS, differentially expressed proteins (DEPs) were also screened with a fold change $\geq 1.2$. Compared with the $\mathrm{CCl}_{4}$-untreated group, 1412 proteins were differentially expressed, of which 668 genes were down-regulated and 744 were up-regulated. In comparison of DEGs and DEPs, 523 gene symbols were overlapped. Wayne analysis based on DEGs and DEPs is shown in Fig. 3.

\section{GO and Pathway analysis}

GO and KEGG pathway analysis were carried out for the overlapped DEGs and DEPs. GO analysis with DAVID indicated that these 523 overlapped proteins were enriched in $182 \mathrm{GO}$ biological process (BP) terms (count $=2$, ease $=0.05$ ) for oxidation reduction, response to extracellular stimulus, response to endogenous stimulus, glucose metabolic process, oxidoreduction coenzyme metabolic process, response to oxidative stress, liver development, cellular response to extracellular stimulus, extracellular matrix organization, extracellular structure organization, and actin filament organization mostly (Fig. 4A).

KEGG pathway analysis indicated that these overlapped proteins were enriched in 36 pathways ( count $=2$, ease $=0.05$ ) which were Drug metabolism, Metabolism of xenobiotics by cytochrome P450, Retinol metabo-

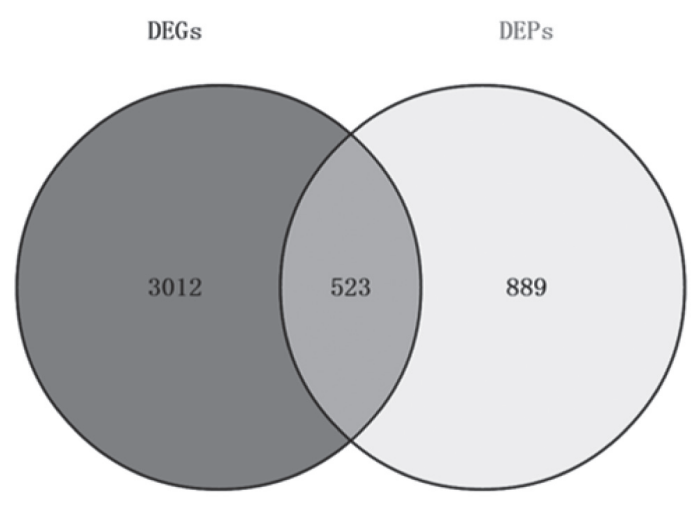

Fig. 3. Wayne analysis based on DEGs and DEPs. Dark part, DEGs; light part, DEPs; the middle part, overlapped part of DEGs and DEPs. lism, PPAR signaling pathway (Peroxisome Proliferator Activated Receptor signaling pathway), Arachidonic acid metabolism, and Glycerolipid metabolism mostly (Fig. 4B).

\section{PPI network}

Based on the overlapped data, a protein-protein interaction (PPI) network was constructed with STRING 9.1. All these proteins were enriched in 2646 pairs interactions (Fig. 5(1)). Then the network was analyzed with Cytoscape 3.2. Degrees and other parameters of the nodes were calculated to screen the hub proteins that may play important roles in the progress of liver damage and liver fibrosis. In this PPI network, 154 proteins with higher degree which was greater than mean degree (mean degree $=5292 / 473=11.19$ ) were considered to work an important regulation effect. The top 10 hub proteins in PPI network were Acly, Cad, ALDH2, Aldh9a1, ALDH7A1, ALDH3A2, ACS11, IFFO2, ALDH1A3 and DPYD (Table 2).

\section{Key function and targets}

In total GO terms, oxidation reduction, response to extracellular stimulus, response to endogenous stimulus, glucose metabolic process, oxidoreduction coenzyme metabolic process, response to oxidative stress, liver development, cellular response to extracellular stimulus, extracellular matrix organization, extracellular structure organization, and actin filament organization were much more important role in the process of $\mathrm{CCl}_{4}$-induced liver fibrosis in rats. And then networks in each GO term were constructed and their related key targets were found (Fig. 5 (2) A, Table 3). The results showed that many targets are involved in multi GO terms (degree $>1$ ). There were much higher degree in Lactate dehydrogenase A (LDHA), Aldolase B, fructose-bisphosphate (ALDOB), Collagen, type I, alpha 1 (COL1A1), Allograft inflammatory factor 1 (AIF1) and Aldehyde dehydrogenase 2 (ALDH2) in the overlapped PPI network.

In total 36 pathways of Drug metabolism, Metabolism of xenobiotics by cytochrome P450, Retinol metabolism, PPAR signaling pathway, Arachidonic acid metabolism, glycolysis/gluconeogenesis, and glycerolipid metabolism 
Mechanisms of $\mathrm{CCl}_{4}$-induced liver fibrosis

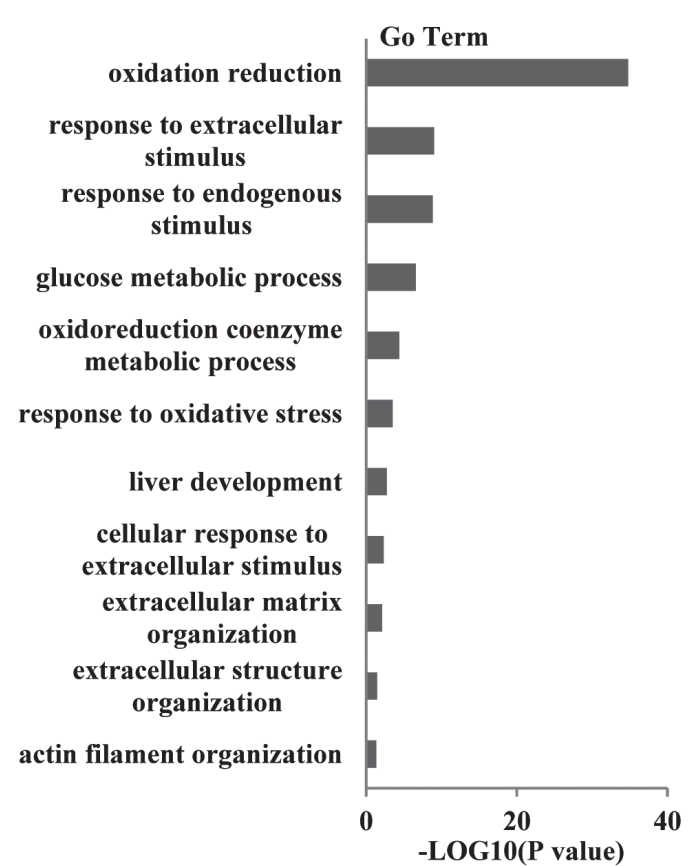

(A)

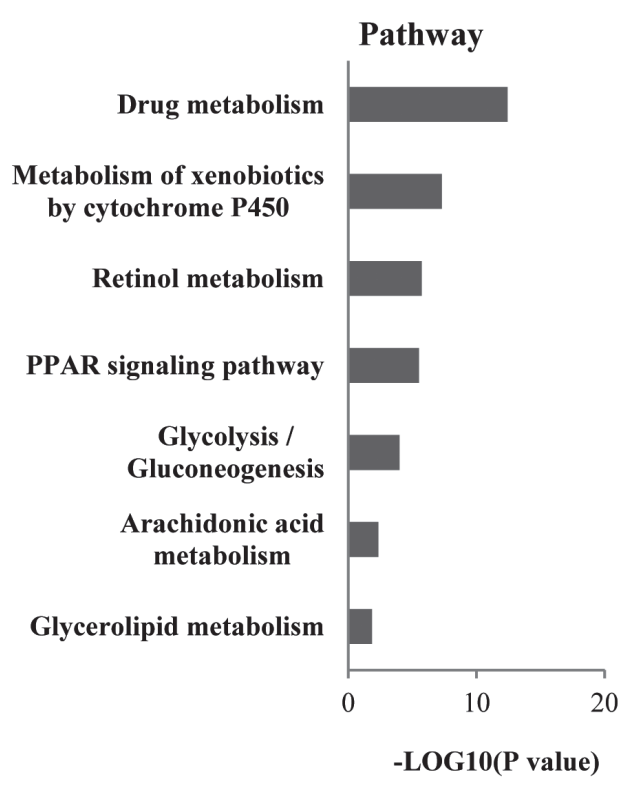

(B)

Fig. 4. Primary biological process terms and pathways enriched by overlapped data between $\mathrm{CCl}_{4}$-untreated and $\mathrm{CCl}_{4}$ treated group. A, key GO_BP Term; B, key KEGG pathway.

Table 2. Top 10 hub proteins in PPI network.

\begin{tabular}{lcccc}
\hline Protein name & Degree & Clustering coefficient & Betweenness centrality & Topological coefficient \\
\hline Acly & 96 & 0.147368 & 0.13096101 & 0.079005 \\
Cad & 72 & 0.125587 & 0.10258738 & 0.078211 \\
ALDH2 & 66 & 0.176224 & 0.03562693 & 0.099624 \\
Aldh9a1 & 62 & 0.199365 & 0.03001064 & 0.103963 \\
ALDH7A1 & 0.208355 & 0.02267156 & 0.106576 \\
Aldh3a2 & 52 & 0.220339 & 0.015402 & 0.109199 \\
Acs11 & 59 & 0.201299 & 0.04179338 & 0.123665 \\
Iffo2 & 56 & 0.296296 & 0.01231337 & 0.121475 \\
Aldh1a3 & 55 & 0.180992 & 0.01691885 & 0.111699 \\
Dpyd & 54 & 0.188679 & 0.03910656 & 0.099906 \\
\hline
\end{tabular}

were much more important role in the process of $\mathrm{CCl}_{4}$-induced liver fibrosis in rats. And then a network of the seven pathways was constructed and their related key targets were found (Fig. 5 (2) B, Table 3). The results showed that Cytochrome P450, family 2, subfamily b, polypeptide 3 (CYP2B3), Cytochrome P450, family 4, subfamily a, polypeptide 3 (CYP4A3), Alcohol dehydrogenase 5 (class III), chi polypeptide (ADH5), Aldehyde dehydrogenase 7 family, member A1 (ALDH7A1) and so on were involved in multi pathways (degree> 1). There was much higher degree in ALDH2, ALDH7A1, Aldehyde dehydrogenase 9 family, member A1(ALDH9A1), Aldehyde dehydrogenase 3 family, member A2 (ALDH3A2), Aldehyde dehydrogenase 1 family, member A3 (ALDH1A3) in the overlapped PPI network.

And we also compared those targets in key function and pathway with liver fibrosis related genes in DrugBank (there are 1740 genes related to liver fibrosis in 


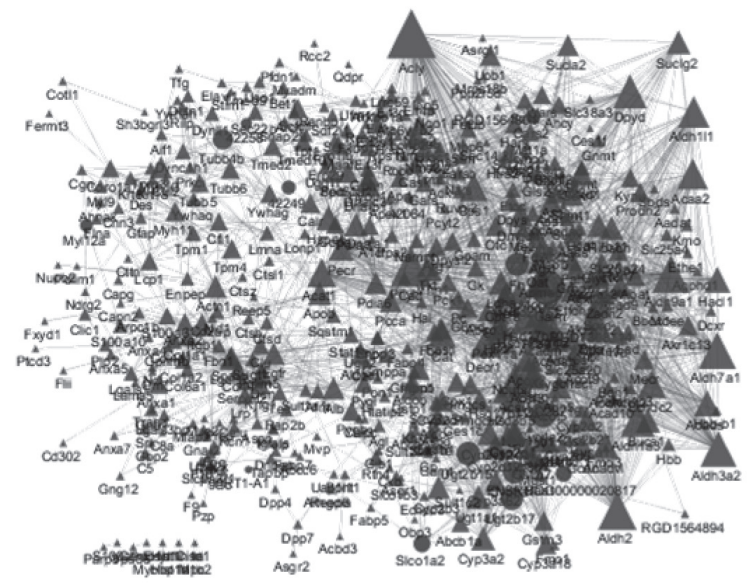

(1)

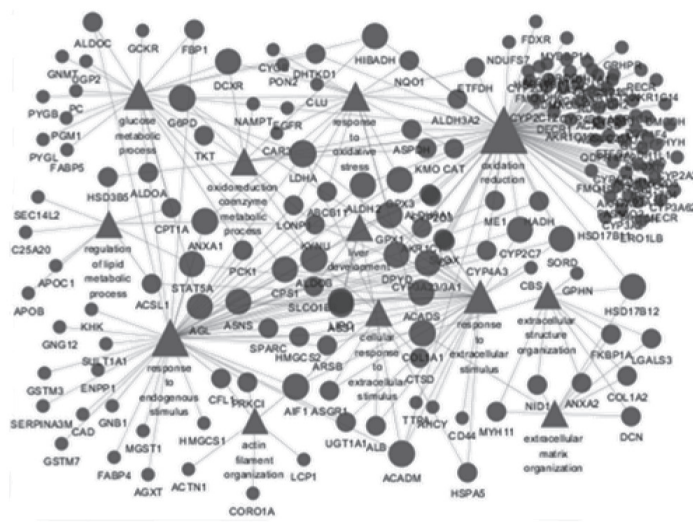

(2)A

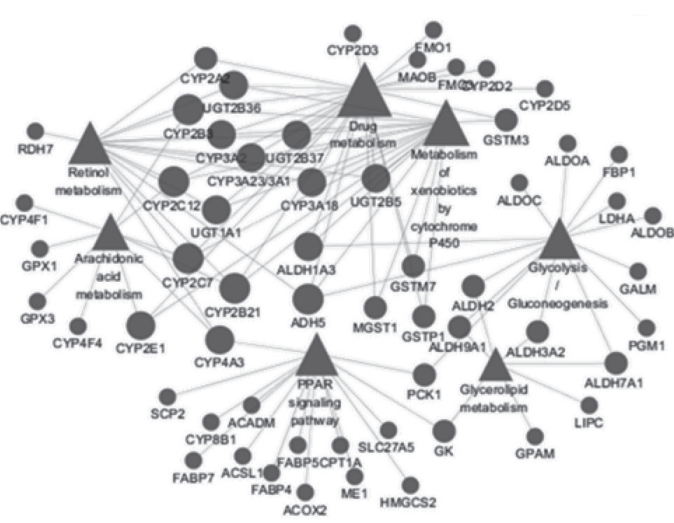

(2)B

Fig. 5. Network of overlapped proteins, key function and their related targets. (1) Network of overlapped proteins between $\mathrm{CCl}_{4}{ }^{-}$ untreated and $\mathrm{CCl}_{4}$-treated group. Triangle nodes, overlapped proteins; Ellipse nodes, interacting with the overlapped proteins in STRING. (2) Network of key function and their related targets. A, network of key GO term and their related targets. Triangle node, GO_term; Ellipse node, related target. B, network of key pathway and their related targets. Triangle node, key pathway; Ellipse node, related target.

DrugBank), 47 targets were overlapped which proved that there was a much close relationship with liver fibrosis in $\mathrm{CCl}_{4}$ toxicity.

\section{Validation of key targets}

In order to verify the results of our analysis, we test the expressions of CYP4A3, ALDH2, ALDH7A1 by Western blot and qRT-PCR (Fig. 6), which had much higher degree in network of important pathways (Table 3). QRT-PCR was carried out using a cohort of independent samples of the $\mathrm{CCl}_{4}$-untreated group $(\mathrm{n}=5)$ and $\mathrm{CCl}_{4}$-treated group $(n=5)$ group. The expressions of CYP4A3, ALDH2 and ALDH7A1 mRNAs were consistent with the results from microarrays. The expression of the three genes decreased in the $\mathrm{CCl}_{4}$-treated group compared to the $\mathrm{CCl}_{4}$-untreated group, although there was no statistical significance. Furthermore, Western blot was also carried out using the same samples. The expressions of CYP4A3, ALDH2 and ALDH7A1 were consistent with the results in QRT-PCR. At the protein expression level, CYP4A3, ALDH2 and ALDH7A1 decreased in the $\mathrm{CCl}_{4}$-treated group compared to the $\mathrm{CCl}_{4}$-untreated group.

\section{DISCUSSION}

$\mathrm{CCl}_{4}$ administration leads to infiltration of the inflammatory cells and liver damage eventually develop to liver fibrosis. The pathological progression of liver fibrosis 
Mechanisms of $\mathrm{CCl}_{4}$-induced liver fibrosis

Table 3. Key function and pathways and their related targets.

\begin{tabular}{lcl}
\hline Key function and pathways & $\begin{array}{c}\text { Numbers of } \\
\text { targets }\end{array}$ & \multicolumn{1}{l}{ Key target (degree) } \\
\hline GO term & & \\
\hline oxidation reduction & 92 & LDHA(7) G6PD(5) ACADM(4) GPX1(4) ALDH2(3) \\
response to extracellular stimulus & 50 & LDHA(7) ALDOB(6) COL1A1(5) ACADM(4) GPX1(4) \\
response to endogenous stimulus & 22 & LDHA(7) ALDOB(6) G6PD(5) AGL(3) CPS1(3) \\
glucose metabolic process & 10 & LDHA(7) ALDOB(6) G6PD(5) DCXR(3) HIBADH(3) \\
oxidoreduction coenzyme metabolic process & 17 & LDHA(7)COL1A1(5) G6PD(5) GPX1(4) GPX3(3) \\
response to oxidative stress & 9 & ALDOB(6) ACADM(4) ALDH2(3) ANXA1(3) ASS1(3) \\
liver development & 8 & LDHA(7) ALDOB(6) AIF1(4) ASNS(3) ASGR1(2) \\
cellular response to extracellular stimulus & 9 & COL1A1(5) HSD17B12(3) LGALS3(2) ANXA2(2) COL1A2(2) \\
extracellular matrix organization & 10 & COL1A1(5) HSD17B12(3) ANXA2(2) COL1A2(2) DCN(2) \\
extracellular structure organization & 6 & AIF1(4) CFL1(2) PRKCI(2) CORO1A(1) ACTN1(1) \\
actin filament organization & & \\
\hline Pathways & 25 & CYP2B3(4) CYP2C7(4) ADH5(4) CYP2B21(4) CYP2C12(4) \\
\hline Drug metabolism & 18 & CYP2B3(4) CYP2C7(4) ADH5(4) CYP2B21(4) CYP2C12(4) \\
Metabolism of xenobiotics by cytochrome P450 & 15 & ADH5(4) CYP2C7(4) CYP2C12(4) CYP2B21(4) CYP2B3(4) \\
Retinol metabolism & 15 & CYP4A3(3) PCK1(2) GK(2) ACADM(1) CYP8B1(1) \\
PPAR signaling pathway & 14 & ADH5(4) ALDH1A3(3) ALDH3A2(2) ALDH7A1(2) ALDH2(2) \\
Glycolysis / Gluconeogenesis & 10 & CYP2B3(4) CYP2C7(4) CYP2C12(4) CYP2B21(4) CYP4A3(3) \\
Arachidonic acid metabolism & 7 & ALDH7A1(2) ALDH2(2) GK(2) ALDH9A1(2) ALDH3A2(2) \\
Glycerolipid metabolism & &
\end{tabular}

A
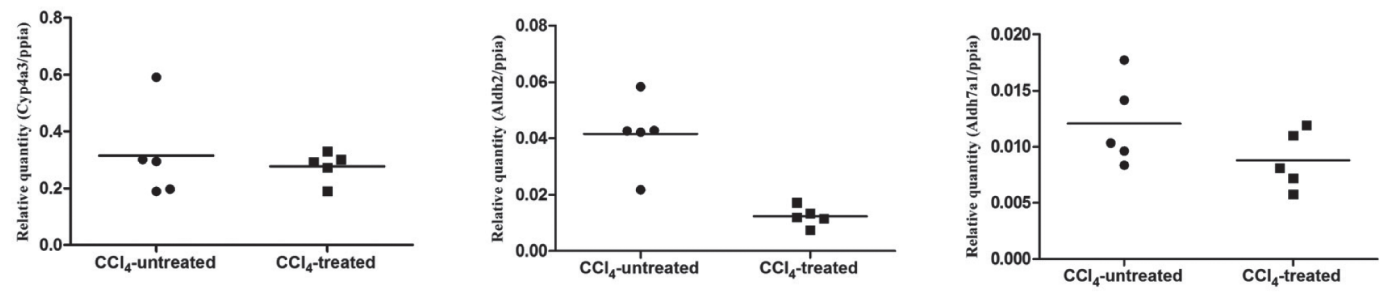

B

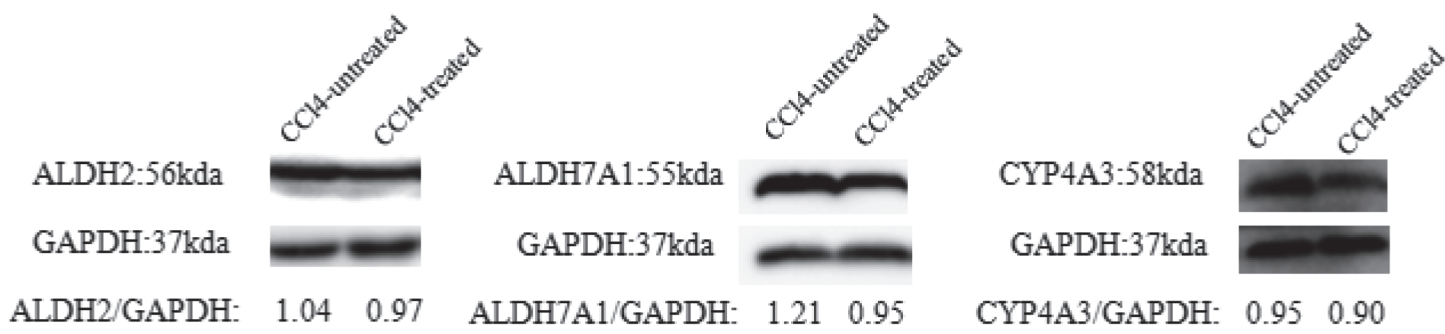

Fig. 6. Expressions of CYP4A3, ALDH2 and ALDH7A1 with qRT-PCR and Western blot. CYP4A3, ALDH2 and ALDH7A1 decreased in $\mathrm{CCl}_{4}$-treated group compared to $\mathrm{CCl}_{4}$-untreated group. A, qRT-PCR. Results were showed with Mean \pm S.D., $\mathrm{n}=5$. B: Western blot. Quality analysis was performed to demonstrate relative quantities in liver tissues, and the ratio of target gene or protein/GAPDH was showed. 
is highly related to the fibrotic response and the proliferation of connective tissue (Guo et al., 2013). In this study, we induced liver lesion and fibrosis using $\mathrm{CCl}_{4}$ in male Wistar rats, performed the integrative analyses of transcriptomics and proteomics with liver fibrosis tissue compared that of $\mathrm{CCl}_{4}$-untreated rat, and identified several important biological process and pathways which enriched by the overlapped differentially expressed genes and proteins.

In this study, the rats treated with $\mathrm{CCl}_{4}$ also exhibited inflammation and fibrosis after 9 weeks injection of $\mathrm{CCl}_{4}$. The body weight and the ratio of liver/body of rats were significantly decreased and ALT, AST were significantly increased in the $\mathrm{CCl}_{4}$-induced liver fibrosis group. HE staining showed there was infiltration of inflammatory cells around the edematous liver cells and from the Sirius red staining, collagen deposition in the liver after the chemical damage was detected. There was higher content of Hyp with significantly difference in the $\mathrm{CCl}_{4}$-induced liver fibrosis group. All the results above suggested that the basic toxicity of $\mathrm{CCl}_{4}$ is to develop inflammatory infiltration and fibrotic hyperplasia in the liver.

Compared with the $\mathrm{CCl}_{4}$-induced liver fibrosis group, 3535 genes and 1412 proteins were differentially expressed. 523 overlapped proteins are screened, which mainly involved in the process of oxidation reduction, response to oxidative stress, extracellular matrix organization and so on. Pathways involved mainly included Retinol metabolism, PPAR signaling pathway, Arachidonic acid metabolism, glycolysis/gluconeogenesis, and glycerolipid metabolism.

Exposure of liver tissues and cells to $\mathrm{CCl}_{4}$ initiates a complex process for resistance to toxicity. $\mathrm{CCl}_{4}$ metabolism in the liver results in the production of free radicals (Sancheti et al., 2013), and triggers oxidative stress which has been considered as a conjoint pathological mechanism, and contributes to initiation and progression of liver injury (Li et al., 2015a). Oxidative stress will further initiate the production of inflammatory cytokines (Heeba and Morsy, 2015), cause necrosis of hepatocytes, induce inflammation, and further promote progression of hepatic fibrogenesis (Heeba and Mahmoud, 2014), which was demonstrated by the increased levels of ALT, AST, Hyp and pathological examinations in this study. And our transcriptomics and proteomics results both indicated that the overlapped genes/proteins involved in process of oxidation reduction and response to oxidative stress, which finally lead to liver injury and fibrosis.

When the liver is subjected to chronic injury, hepatic stellate cells (HSCs) are activated and proliferate, causing ECM deposition, and hepatocytes are replaced with abun- dant ECM (Duval et al., 2014) which leads to scar formation and fibrosis (Roy et al., 2015), which were proved in Sirius red staining. ECM-involved signaling was also reported to be considered a paracrine/endocrine function, as it affects cell phenotype, function, fate, and finally tissue homeostasis (Karsdal et al., 2015). Additionally, the process of glucose metabolism and liver development were found to be involved in the toxicity of $\mathrm{CCl}_{4}$. The liver is a central organ in gluconeogenesis metabolism (Bechmann et al., 2012). Usually after liver injury, there would be glucose metabolic disorder (Bahr et al., 2006) and abnormal liver development (Yin et al., 2013). According to our discussion above, we figured out that the toxicity of $\mathrm{CCl}_{4}$ may be associated with many biological processes.

According to the results of pathway analysis, we found that the toxicity of $\mathrm{CCl}_{4}$ involved in multi-pathways. Retinol and its derivative, retinoic acid, have critical roles in liver regeneration and pathogenesis including inflammation, steatosis, fibrosis, cirrhosis and cancer (Park et al., 2012). Retinol as well as cellular retinoic acid binding proteins and retinol-metabolizing enzymes are stored in HSCs (Miyazaki et al., 2014). During the fibrotic process, hepatic stellate cells are activated and lose their lipid drops which store retinol (de Paula et al., 2010). Impaired retinol status was reported to be associated with development of cirrhosis (Chang et al., 2008). In this study, we found retinol pathway involved in the development of liver fibrosis induced by $\mathrm{CCl}_{4}$. CYP4A3, involved in retinol pathway decreased in the $\mathrm{CCl}_{4}$ treated group and it will change the levels of retinol and retinoic acid which may further affect the action of HSCs (Lee et al., 2015) and finally develop into liver fibrosis.

PPAR signaling pathway and Arachidonic acid metabolism also played a key role in liver injury and fibrosis induced by $\mathrm{CCl}_{4}$. In activated HSCs, activity of PPAR- $\gamma$ is decreased, which leads to trans-differentiation of HSCs from the retinoid storing state to the ECM-producing myofibroblasts (Kumar et al., 2014). The activation of PPAR- $\gamma$ was shown to have a relationship with expression of collagen type I induced by TGF- $\beta 1$ (Bian et al., 2013) and inhibit hepatic stellate cell activation and progression of fibrosis (Attia et al., 2013). Studies have indicated that arachidonic acid is involved in oxidative stress and in inflammatory response (Holownia et al., 2014; Le et al., 2012; Xie et al., 2015) which was also been proved in our GO analysis. ALDH2 and ALDH7A1 were involved in glycolysis/Gluconeogenesis and glycerolipid metabolism, and the results of qRT-PCR and Western blot showed that the expression of ALDH2 and ALDH7A1 decreased after liver injury. Speaking of glycolysis, in chronic liv- 
Mechanisms of $\mathrm{CCl}_{4}$-induced liver fibrosis

er injury, energy production will change from mitochondrial respiration to glycolysis which plays a key role in energy metabolism in the liver (Nishikawa et al., 2014). The liver is the main organ for the metabolism of glucose and lipid (Bou Khalil et al., 2010; Hammond et al., 2002) and when lesion occurs, the liver may dysfunction including abnormal glycolysis/gluconeogenesis and glycerolipid metabolism (Prentki and Madiraju, 2008; Dwyer et al., 2012).

In targets we screened in key function and key pathway, 47 targets were related to liver fibrosis based on DrugBank searches. The results indicated that the toxicity of $\mathrm{CCl}_{4}$ to induce liver fibrosis from the perspective of the transcriptomic changes, which was also consistent with our histopathology and biochemical test. The targets which showed no relationship with liver fibrosis in DrugBank may play an important role in other reactions caused by the toxicity of $\mathrm{CCl}_{4}$, which needs further investigations. Moreover, the further validation of our findings based on microarray and iTRAQ analysis is also important in the future.

In conclusion, the toxicity of $\mathrm{CCl}_{4}$ to induce liver lesion and liver fibrosis had already been fully investigated, while the studies for molecular mechanism were far from enough. In this study, we performed mRNA microarray and ITRAQ analysis to investigate the molecular mechanism of $\mathrm{CCl}_{4}$ toxicity which may clarify the toxicological mechanism and lead to better detoxification. This study showed that when the liver was exposed to $\mathrm{CCl}_{4}$, it mobilized different kinds of biological processes, multi-targets and multi-pathways, including Retinol metabolism, PPAR signaling pathway, Arachidonic acid metabolism, glycolysis/gluconeogenesis, and glycerolipid metabolism by regulating targets such as CYP4A3, ALDH7A1 and ALDH2. However, the specific mechanism still needs further research and the analysis results need experiments to be verified.

\section{ACKNOWLEDGMENTS}

This work was supported by Key Program of National Natural Science Foundation of China (81330084), Shanghai Municipal Science and Technology Commission Project (12401900401), E-institutes of Shanghai Municipal Education Commission (E03008).

Conflict of interest---- The authors declare that there is no conflict of interest.

\section{REFERENCES}

Attia, Y.M., Elalkamy, E.F., Hammam, O.A., Mahmoud, S.S. and El-hatib, A.S. (2013): Telmisartan, an AT1 receptor blocker and a PPAR gamma activator, alleviates liver fibrosis induced experimentally by Schistosoma mansoni infection. Parasit. Vectors, $\mathbf{6}$, 199.

Bahr, M.J., Ockenga, J., Böker, K.H., Manns, M.P. and Tietge, U.J. (2006): Elevated resistin levels in cirrhosis are associated with the proinflammatory state and altered hepatic glucose metabolism but not with insulin resistance. Am. J. Physiol. Endocrinol. Metab., 291, E199-206.

Bechmann, L.P., Hannivoort, R.A., Gerken, G., Hotamisligil, G.S., Trauner, M. and Canbay, A. (2012): The interaction of hepatic lipid and glucose metabolism in liver diseases. J. Hepatol., 56, 952-964.

Bian, D., Zhang, J., Wu, X., Dou, Y., Yang, Y., Tan, Q., Xia, Y., Gong, Z. and Dai, Y. (2013): Asiatic Acid Isolated From Centella Asiatica Inhibits TGF- $\beta 1$-induced Collagen Expression in Human Keloid Fibroblasts via PPAR- $\gamma$ Activation. Int. J. Biol. Sci., 9, 1032-1042.

Bou Khalil, M., Blais, A., Figeys, D. and Yao, Z. (2010): Lipin The bridge between hepatic glycerolipid biosynthesis and lipoprotein metabolism. Biochim. Biophys. Acta, 1801, 1249-1259.

Chang, W.T., Ker, C.G., Hung, H.C., Lee, K.T., Chen, L.S., Chiang, H.C. and Huang, M.C. (2008): Albumin and prealbumin may predict retinol status in patients with liver cirrhosis. Hepatogastroenterology, 55, 1681-1685.

de Paula, T.P., Ramalho, A. and Braulio, V.B. (2010): The effectiveness of relative dose response to retinol intake as an evaluation of vitamin A status of cirrhotic patients. J. Hum. Nutr. Diet., 23, 583-589.

Dong, S., Chen, Q.L., Feng, Q., Hu, Y.Y., Liu, P. and Su, S.B. (2015): Study on molecular mechanism of improving rat liver fibrosis with Fuzheng Huayu Formula based on gene profile. CJTCMP, 30, 1812-1817.

Duval, F., Moreno-Cuevas, J.E., González-Garza, M.T., Maldonado-Bernal, C. and Cruz-Vega, D.E. (2015): Liver fibrosis and mechanisms of the protective action of medicinal plants targeting inflammation and the immune response. Int. J. Inflam., 2015, 943497.

Duval, F., Moreno-Cuevas, J.E., González-Garza, M.T., Rodríguez-Montalvo, C. and Cruz-Vega, D.E. (2014): Protective mechanisms of medicinal plants targeting hepatic stellate cell activation and extracellular matrix deposition in liver fibrosis. Chin. Med., 9, 27.

Dwyer, J.R., Donkor, J., Zhang, P., Csaki, L.S., Vergnes, L., Lee, J.M., Dewald, J., Brindley, D.N., Atti, E., Tetradis, S., Yoshinaga, Y., De Jong, P.J., Fong, L.G., Young, S.G. and Reue, K. (2012): Mouse lipin-1 and lipin-2 cooperate to maintain glycerolipid homeostasis in liver and aging cerebellum. Proc. Natl. Acad. Sci. USA, 109, E2486- E2495.

Ebeid, H.M., Gibriel, A.A., Al-Sayed, H.M., Elbehairy, S.A. and Motawe, E.H. (2015): Hepatoprotective and antioxidant effects of wheat, carrot, and mango as nutraceutical agents against $\mathrm{CCl}_{4}^{-}$ induced hepatocellular toxicity. J. Am. Coll. Nutr., 34, 228-231.

El-Bini, Dhouib.I., Lasram, M.M., Annabi, A., Gharbi, N. and El-Fazaa, S. (2015): A comparative study on toxicity induced by carbosulfan and malathion in Wistar rat liver and spleen. Pestic. Biochem. Physiol., 124, 21-28.

Enomoto, M., Morikawa, H., Tamori, A. and Kawada, N. (2014): 
Noninvasive assessment of liver fibrosis in patients with chronic hepatitis B. World J. Gastroenterol., 20, 12031-12038.

Guo, C., Xu, L., He, Q., Liang, T., Duan, X. and Li, R. (2013): Anti-fibrotic effects of puerarin on CCl4-induced hepatic fibrosis in rats possibly through the regulation of PPAR- $\gamma$ expression and inhibition of PI3K/Akt pathway. Food Chem. Toxicol., 56, 436-442.

Hammond, L.E., Gallagher, P.A., Wang, S., Hiller, S., Kluckman, K.D., Posey-Marcos, E.L., Maeda, N. and Coleman, R.A. (2002): Mitochondrial glycerol-3-phosphate acyltransferase-deficient mice have reduced weight and liver triacylglycerol content and altered glycerolipid fatty acid composition. Mol. Cell Biol., 22, 8204-8214.

Heeba, G.H. and Mahmoud, M.E. (2014): Therapeutic potential of morin against liver fibrosis in rats: modulation of oxidative stress, cytokine production and nuclear factor kappa B. Environ Toxicol. Pharmacol., 37, 662-671.

Heeba, G.H. and Morsy, M.A. (2015): Fucoidan ameliorates steatohepatitis and insulin resistance by suppressing oxidative stress and inflammatory cytokines in experimental non-alcoholic fatty liver disease. Environ. Toxicol. Pharmacol., 40, 907-914.

Holownia, A, Mroz, R.M., Wielgat, P., Jakubow, P., Jablonski, J., Sulek, J. and Braszko, J.J. (2014): Histone acetylation and arachidonic acid cytotoxicity in HepG2 cells overexpressing CYP2E1. Naunyn Schmiedebergs Arch. Pharmacol., 387, 271280.

Huang, Z.G., Zhai, W.R., Zhang, Y.E. and Zhang, X.R. (1998): Study of heteroserum-induced rat liver fibrosis model and its mechanism. World J. Gastroenterol., 4, 206-209.

Karsdal, M.A., Manon-Jensen, T., Genovese, F., Kristensen, J.H., Nielsen, M.J., Sand, J.M., Hansen, N.U., Bay-Jensen, A.C., Bager, C.L., Krag, A., Blanchard, A., Krarup, H., Leeming, D.J. and Schuppan, D. (2015): Novel insights into the function and dynamics of extracellular matrix in liver fibrosis. Am. J. Physiol. Gastrointest Liver Physiol., 308, G807-830.

Kong, X., Zhou, W., Wan, J.B., Zhang, Q., Ni, J. and Hu, Y. (2015): An Integrative Thrombosis Network: Visualization and Topological Analysis. Evid. Based Complement. Alternat. Med., 2015, 265303.

Kumar, V., Mundra, V. and Mahato, R.I. (2014): Nanomedicines of Hedgehog inhibitor and PPAR- $\gamma$ agonist for treating liver fibrosis. Pharm. Res., 31, 1158-1169.

Le, H.D., Meisel, J.A., de Meijer, V.E., Fallon, E.M., Gura, K.M., Nose, V., Bistrian, B.R. and Puder, M. (2012): Docosahexaenoic acid and arachidonic acid prevent essential fatty acid deficiency and hepatic steatosis. JPEN J. Parenter. Enteral. Nutr., 36, 431441.

Lee, H., Jeong, H., Park, S., Yoo, W., Choi, S., Choi, K., Lee, M.G., Lee, M., Cha, D., Kim, Y.S., Han, J., Kim, W., Park, S.H. and Oh, J. (2015): Fusion protein of retinol-binding protein and albumin domain III reduces liver fibrosis. EMBO Mol. Med., 7, 819830 .
Li, S., Tan, H.Y., Wang, N., Zhang, Z.J., Lao, L., Wong, C.W. and Feng, Y. (2015): The role of oxidative stress and antioxidants in liver diseases. Int. J. Mol. Sci., 16, 26087-26124.

Li, Z.M., Sun, J.H. and Yang, X.M. (2015): Recent advances in molecular magnetic resonance imaging of liver fibrosis. Biomed. Res. Int., 2015, 595467.

Meierhofer, D., Weidner, C. and Sauer, S. (2014): Integrative analysis of transcriptomics, proteomics, and metabolomics data of white adipose and liver tissue of high-fat diet and rosiglitazonetreated insulin-resistant mice identified pathway alterations and molecular hubs. J. Proteome Res., 13, 5592-5602.

Miyazaki, H., Takitani, K., Koh, M., Inoue, A., Kishi, K. and Tamai, H. (2014): Retinol statue and expression of retinol-related proteins in methionine-choline deficient rats. J. Nutr. Sci. Vitaminol., 60, 78-85.

Nishikawa, T., Bellance, N., Damm, A., Bing, H., Zhu, Z., Handa, K., Yovchev, M.I., Sehgal, V., Moss, T.J., Oertel, M., Ram, P.T., Pipinos, I.I., Soto-Gutierrez, A., Fox, I.J. and Nagrath, D. (2014): A switch in the source of ATP production and a loss in capacity to perform glycolysis are hallmarks of hepatocyte failure in advance liver disease. J. Hepatol., 60, 1203-1211.

Osada, J. (2013): The use of transcriptomics to unveil the role of nutrients in mammalian liver. ISRN Nutr., 2013, 403792.

Park, S., Choi, S., Lee, M.G., Lim, C. and Oh, J. (2012): Retinol binding protein-albumin domain III fusion protein deactivates hepatic stellate cells. Mol Cells., 34, 517-522.

Prentki, M. and Madiraju, S.R. (2008): Glycerolipid metabolism and signaling in health and disease. Endocr Rev., 29, 647-676.

Roy, S., Benz, F., Vargas Cardenas, D., Vucur, M., Gautheron, J., Schneider, A., Hellerbrand, C., Pottier, N., Alder, J., Tacke, F., Trautwein, C., Roderburg, C. and Luedde, T. (2015): miR-30c and miR-193 are a part of the TGF- $\beta$-dependent regulatory network controlling extracellular matrix genes in liver fibrosis. J. Dig. Dis., 16, 513-524.

Sahreen, S., Khan, M.R., Khan, R.A. and Alkreathy, H.M. (2014): Cardioprotective role of leaves extracts of Carissa opaca against $\mathrm{CCl}_{4}$ induced toxicity in rats. BMC Res Notes., 7, 224.

Sancheti, S., Sancheti, S. and Seo, S.Y. (2013): Ameliorative effects of 7-methylcoumarin and 7-methoxycoumarin against $\mathrm{CCl}_{4}$-induced hepatotoxicity in rats. Drug Chem. Toxicol., 36, 42-47.

Xie, C., Li, X., Wu, J., Liang, Z., Deng, F., Xie, W., Zhu, M., Zhu, J., Zhu, W., Geng, S. and Zhong, C. (2015): Anti-inflammatory activity of magnesium isoglycyrrhizinate through inhibition of phospholipase A2/arachidonic acid pathway. Inflammation, 38, 1639-1648.

Xuan, J., Chen, S., Ning, B., Tolleson, W.H. and Guo, L. (2015): Development of HepG2-derived cells expressing cytochrome $\mathrm{P} 450$ s for assessing metabolism-associated drug-induced liver toxicity. Chem Biol Interact., pii: S0009-2797(15)30088-0.

Yin, C., Evason, K.J., Asahina, K. and Stainier, D.Y. (2013): Hepatic stellate cells in liver development, regeneration, and cancer. J. Clin. Invest., 123, 1902-1910. 